Check for updates

Cite this: RSC Adv., 2017, 7, 53164

\title{
Bimetallic gold core-silver shell nanorod performance for surface enhanced Raman spectroscopy $\dagger$
}

\author{
Zeid A. Nima, (D) $\ddagger^{* a}$ Yevgeniy R. Davletshin, (DD $\sharp \xi^{\mathrm{b}}$ Fumyia Watanabe, (D) \\ Karrar M. Alghazali, ${ }^{a}$ J. Carl Kumaradas (D) ${ }^{b}$ and Alexandru S. Biris ${ }^{a}$
}

\begin{abstract}
Plasmonic gold nanorods (AuNRs) coated with four different thickness silver shells (AuNR\Ags) were synthesized and tested for their efficiency in Surface Enhanced Raman Scattering (SERS) signal enhancement for biomedical applications. Both AuNRs and AuNR\Ags were prepared using a modified seed-mediated method and then characterized using TEM, XPS and UV-vis spectroscopy. All four bimetallic nanorods used in our experiments started from gold nanorod (AuNR) cores (of $36 \mathrm{~nm}$ length and $12 \mathrm{~nm}$ diameter) which were coated with a 0,1,2, 3 or $4 \mathrm{~nm}$ thick layer of silver. SERS spectra were obtained for each thickness of AuNR\Ag Raman agent using a Raman reporter - organic molecule $p$-aminothiophenol (PATP). Moreover, to confirm experimental findings a numerical model was built using COMSOL Multiphysics and solved for a single AuNR\Ag interaction with light on a silica substrate. The highest SERS signal at the incident wavelength of $784 \mathrm{~nm}$, was observed for AuNR\Ags coated with a $1 \mathrm{~nm}$ thick silver shell. The numerical model confirmed experimental findings and predicted the highest near-field enhancement in the vicinity of nanoparticles on top of a silica substrate at $784 \mathrm{~nm}$ wavelength, for an AuNR\Ag with the same $1 \mathrm{~nm}$ silver shell thickness.
\end{abstract}

Received 12th June 2017

Accepted 18th October 2017

DOI: $10.1039 / \mathrm{c} 7 \mathrm{ra0} 6573 f$

rsc.li/rsc-advances thousands of times by excitation from the LSPR of a nanoparticle. Optimizing the design of SERS Raman agents is crucial for achieving the largest enhancement possible. ${ }^{1,18-21}$ Historically, SERS Raman agents have taken many different forms ${ }^{22-25}$ starting with roughened metallic surfaces, ${ }^{26}$ colloidal metallic suspensions, ${ }^{27,28}$ thin metal films, and nanoparticles with different shapes. ${ }^{29-31}$ Recently, a combination of these forms and hybrid techniques have been investigated to optimize SERS substrates. ${ }^{32-34}$ Generally silver coated gold nanoparticles have been used recently to enhance Raman signals for several applications..$^{35-40}$

In this work, a new class of SERS agent, bimetallic plasmon nanorods, was designed and synthesized for the use in biomedical applications. Bare gold nanorods (AuNRs) and gold nanorods coated with 1, 2, 3 and $4 \mathrm{~nm}$ thick silver shells (AuNR $\backslash$ Ags) were synthesized using a modified seed mediated method $^{6}$ and characterized using transmission electron microscopy (TEM) and X-ray photoelectron spectroscopy (XPS). LSPR frequencies for each type of AuNR and AuNR $\backslash \mathrm{Ag}$ colloidal solution were determined using UV-vis spectroscopy. The SERS efficiency was determined using $p$-aminothiophenol, a thiolated organic molecule, as the Raman reporter probe. ${ }^{6}$ Finally, for the verification of the results, a finite element model was used to calculate the optical response of a single AuNR and AuNR $\backslash \mathrm{Ag}$ during Raman spectroscopy. The findings from the numerical model were in agreement with the experimental data. 


\section{Experimental}

\subsection{Materials}

The following chemicals were purchased from Sigma-Aldrich and used without further purification: gold(III) chloride trihydrate (99\%), sodium borohydride (99\%), L-ascorbic acid (98\%), $p$-aminothiophenol (PATP), and polyvinylpyrrolidone (PVP) (MW 10 000). Silver nitrate was purchased from Fisher Scientific, and hexadecyltrimethylammonium bromide (CTAB 99\%) from MP Biomedicals.

\subsection{Synthesis of gold nanorods}

Gold nanorods (AuNRs) were synthesized using the seedmediated method developed by Nikoobakht.6,41 A seed solution was first prepared by mixing $5 \mathrm{ml}$ of CTAB solution $(0.2 \mathrm{M})$ with $5 \mathrm{ml}$ of $\mathrm{HAuCl}_{4}(0.0005 \mathrm{M})$, after that $0.6 \mathrm{ml}$ of sodium borohydride $(0.01 \mathrm{M})$ was added, and the solution was stirred for 2-5 minutes. Preparing AuNRs with an aspect ratio of around 3 required the following procedure: $5 \mathrm{ml}$ of CTAB $(0.2$ M) was mixed thoroughly with $150 \mu \mathrm{l}$ of $\mathrm{AgNO}_{3}$ solution (0.004 $\mathrm{M})$, and then $5 \mathrm{ml}$ of $\mathrm{HAuCl}_{4}(0.001 \mathrm{M})$ was added and mixed. Afterwards, $70 \mu$ l of ascorbic acid $(0.0788 \mathrm{M})$ was mixed with the solution, and finally, $12 \mu \mathrm{l}$ of the seed solution was added. The mixed solution was kept at $30{ }^{\circ} \mathrm{C}$ for 40 minutes without any further stirring. The AuNRs were purified several times using a centrifuge at $12000 \mathrm{rpm}$ for $30 \mathrm{~min}$ to remove any chemicals.

\subsection{Coating of AuNRs with a silver shell}

Detailed coating procedures can be found in our previous work. ${ }^{6}$ Purified AuNRs were dispersed in $5 \mathrm{ml}$ CTAB solution, and then $5 \mathrm{ml}$ of $1 \%$ polyvinylpyrrolidone solution was added. Silver nitrate solution (0.001 M) was added in different amounts to achieve multiple shell thickness; 200, 250, 300, and $350 \mu \mathrm{l}$ were added with gentle mixing. Afterwards, $100 \mu \mathrm{l}$ of ascorbic acid (0.1 M) was added, and then $200 \mu \mathrm{l}$ of sodium hydroxide solution $(0.1 \mathrm{M})$ was added to increase the $\mathrm{pH}$ to about 9 . The silver ion reduction reaction required an alkaline medium to begin depositing on the AuNRs surface. Silver-coated gold nanorods (AuNR $\backslash$ Ags) were purified several times using a centrifuge at $10000 \mathrm{rpm}$ and redispersed in deionized water to remove any excess reagents.

\subsection{Assembling SERS probes on AuNR $\backslash A g$}

A small organic molecule was self-assembled on the surface of the AuNRs and AuNR\Ags. p-Aminothiophenol (PATP) molecules were prepared with $10 \mathrm{mM}$ in absolute ethanol stock solution. $5 \mu \mathrm{l}$ of PATP was added to a solution that had $5 \mathrm{ml}$ of AuNR $\backslash \mathrm{Ag}$ and kept under stirring for 3 hours at $45^{\circ} \mathrm{C}$. Any excess materials were removed using a centrifuge for $30 \mathrm{~min}$.

\subsection{Transmission electron microscopy (TEM)}

The morphology and size of the AuNRs and AuNR\Ags were determined using a Transmission Electron Microscope (TEM),

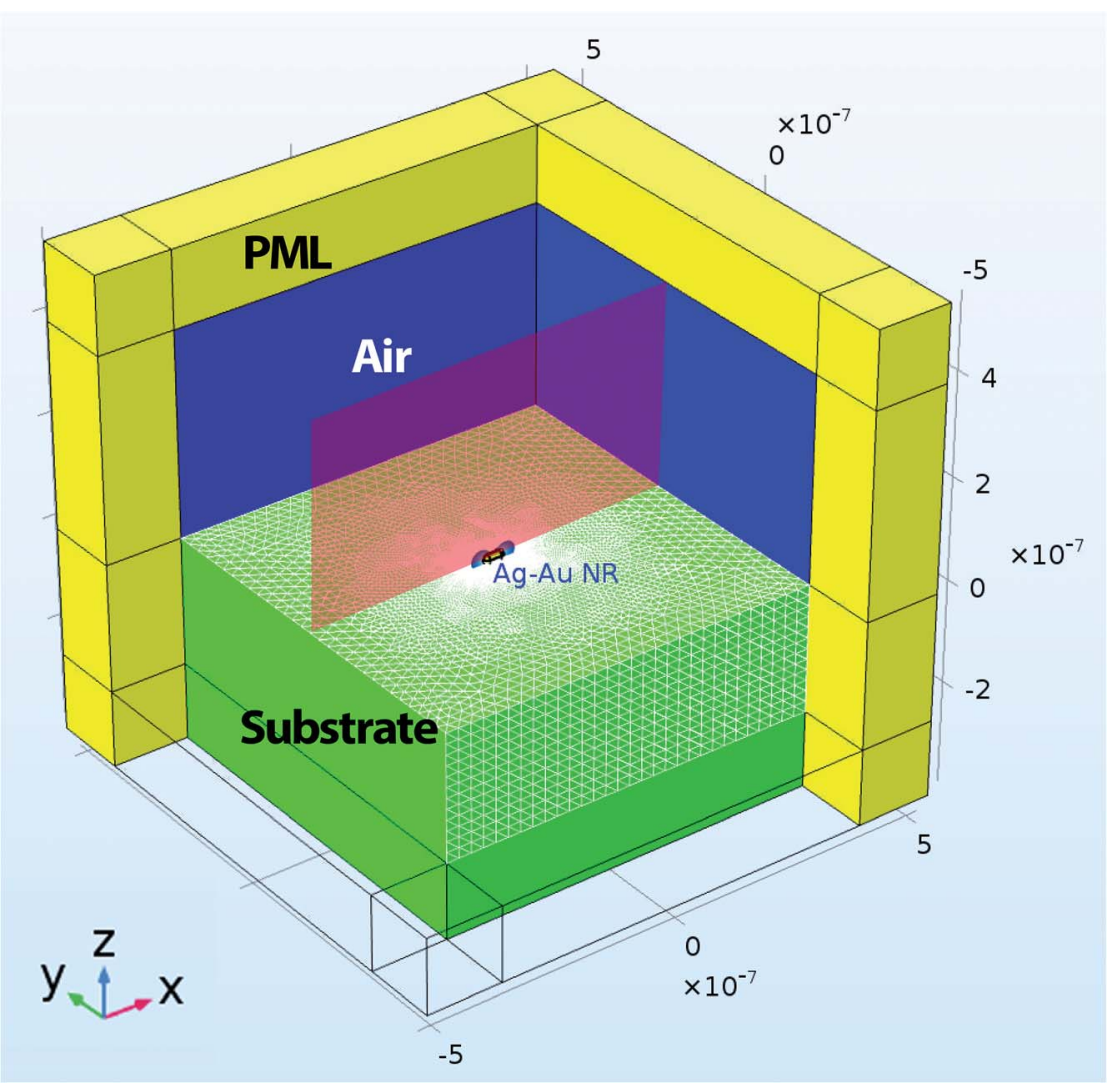

Fig. 1 Schematics of the finite element model. The geometry setup is similar to one that was described in ref. 42. The yellow domain represents perfect matched layers (PML). The green domain shows a silica substrate. The blue domain represents air. The red cross-section plane shows the near field enhancement map with a nanoparticle labelled as AuNR\Ag. 
JEM-2100F (JEOL USA, Peabody, Massachusetts, USA) with an accelerating voltage of $80 \mathrm{kV}$. Each sample was suspended in ethanol and a few drops of the suspension were deposited on holey-carbon coated copper grids, which were allowed to dry for 15 minutes. The average nanorod size and the size distribution of each sample were determined by using Image software (NIH). The TEM was also equipped with an EDAX genesis energy dispersive spectroscopy (EDS) X-ray detection system for spectroscopic analysis.

\subsection{UV-vis-NIR spectra}

For each class of the prepared AuNR $\backslash$ Ags and AuNRs, UV-vis-NIR spectra were recorded in the range between 400-900 nm using a Shimadzu double beam spectrophotometer UV-3600 (UVvisible-NIR) to record electronic spectra. The spectra were collected for aqueous solutions $\left(\sim 0.5 \mathrm{mg} \mathrm{ml}^{-1}\right)$ and then normalized to the highest absorption intensity.

\subsection{SERS spectra}

A few drops of each type of AuNR $\backslash$ Ags and AuNRs, were dried on microscope glass and left under vacuum for three days. After that, each sample was scanned from 400-1700 $\mathrm{cm}^{-1}$ using a Horiba Raman instrument (Horiba Jobin Yvon LabRam
HR800, Edison, New Jersey) assembled by He-Ne laser (17 mW) with a wavelength of $784 \mathrm{~nm}$ and three Olympus BX-51 lenses with a $100 \times$ micro-objectives lens connected to a CCD camera. The spectra were collected using 600-lines per mm gratings with the same acquisition time. The spectrometer has a threedimensional (3-D) $(x-y-z)$ automatic adjustable stage. In all of the measurements, the Raman spectrometer was calibrated using the Si-Si Raman signal located at $521 \mathrm{~cm}^{-1}$ Raman shift. The collected spectral data were smoothed and baselined to represent the final results.

\subsection{Numerical model}

The numerical model was built using commercially available finite element software - COMSOL Multiphysics, version 5.2. The base model of a nanorod on a substrate interaction with an electromagnetic field is similar to an example given in the COMSOL library. ${ }^{42}$ A schematic of the model's geometry is shown in Fig. 1.

An electromagnetic wave with circular polarization was travelling in the negative $Z$-direction (see Fig. 1). A rectangular domain with $1.05 \mu \mathrm{m}$ width and depth and $9.5 \mu \mathrm{m}$ height was used to model a nanorod in the air domain on top of the substrate domain. The dimensions of the AuNR $\backslash$ Ags and AuNRs

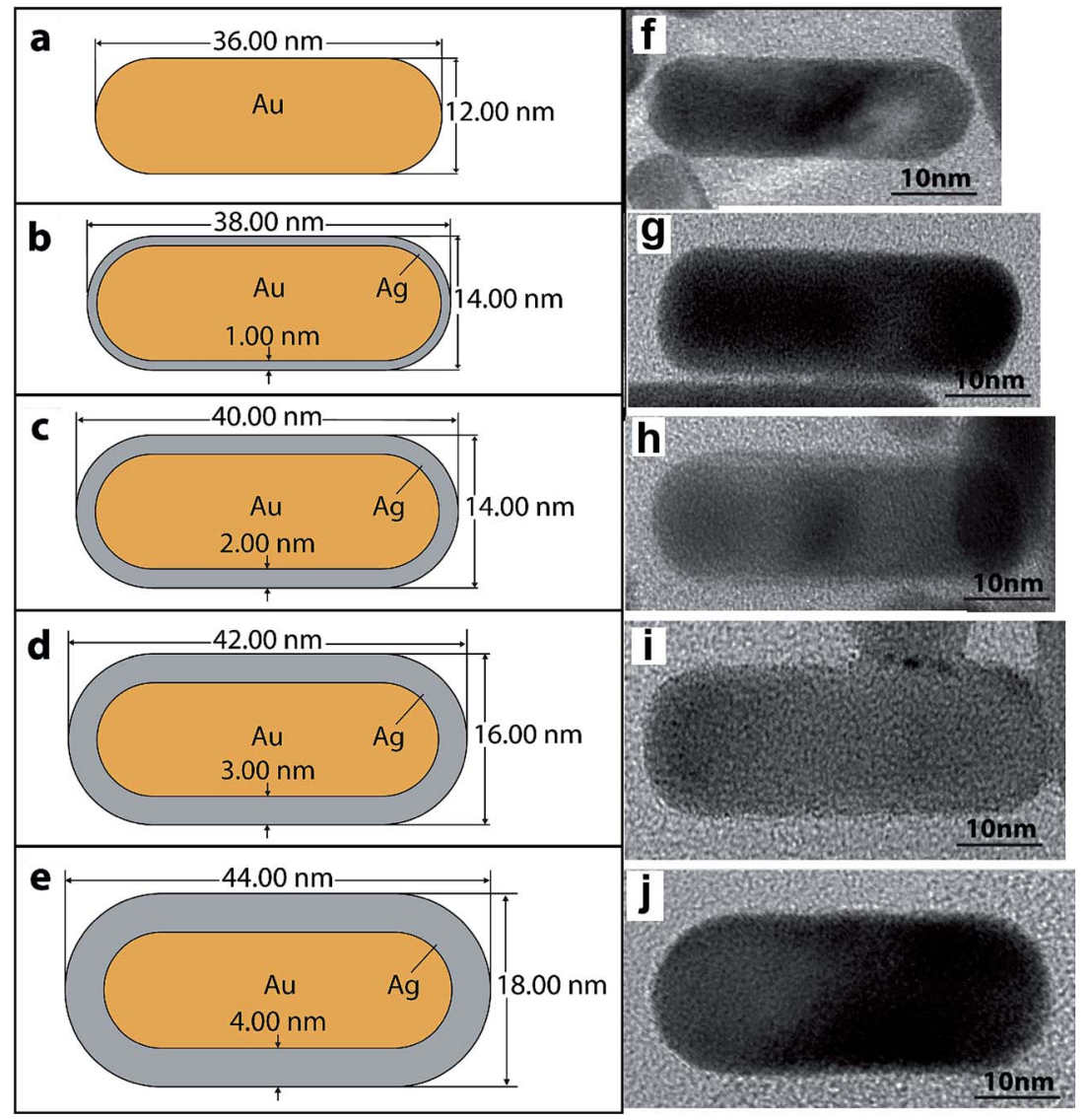

Fig. 2 The dimensions of the AuNRs and AuNR\Ags with $0,1,2,3$, and $4 \mathrm{~nm}$ silver shells and $12 \times 36 \mathrm{~nm}$ gold cores that were used in the numerical model, (a-e) respectively. ( $f-j)$ HRTEM images: $(f)$ a gold nanorod only, and ( $g-j)$ gold nanorods covered with (1, 2, 3, and 4 nm) silver shells, respectively. 
are given in Fig. 2a-e. The air domain had $4.5 \mu \mathrm{m}$ height while the silica substrate was modeled with a height of $4 \mu \mathrm{m}$. The silica substrate was modeled with a bulk refractive index of 1.45. The dielectric constant of the core gold nanorod was defined using Drude formalism with size-corrections to the bulk dielectric function of gold. ${ }^{43}$

$$
\begin{aligned}
\varepsilon_{\mathrm{au}}(\omega, r)= & \varepsilon_{\text {bulk }}(\omega)+\frac{\omega_{\mathrm{p}}^{2}}{\omega^{2}+i \omega \gamma_{0}} \\
& -\frac{\omega_{\mathrm{p}}^{2}}{\omega^{2}+i \omega\left(\gamma_{0}+A_{\mathrm{au}} \frac{v_{\mathrm{f}}}{l_{\mathrm{eff}}}+\gamma_{\mathrm{rad}} V_{\mathrm{au}}\right)},
\end{aligned}
$$

where $\varepsilon_{\text {bulk }}$ is the gold's bulk dielectric function obtained from Johnson and Christy ${ }^{44}$ and $\omega_{\mathrm{p}}=9.096 \mathrm{eV}$ is the bulk plasma frequency. $A_{\mathrm{au}}=1.4$ is the broadening parameter size, ${ }^{45} l_{\mathrm{eff}}$ is the effective confinement length, ${ }^{45} v_{\mathrm{f}}$ is the Fermi velocity, $\gamma_{0}=$
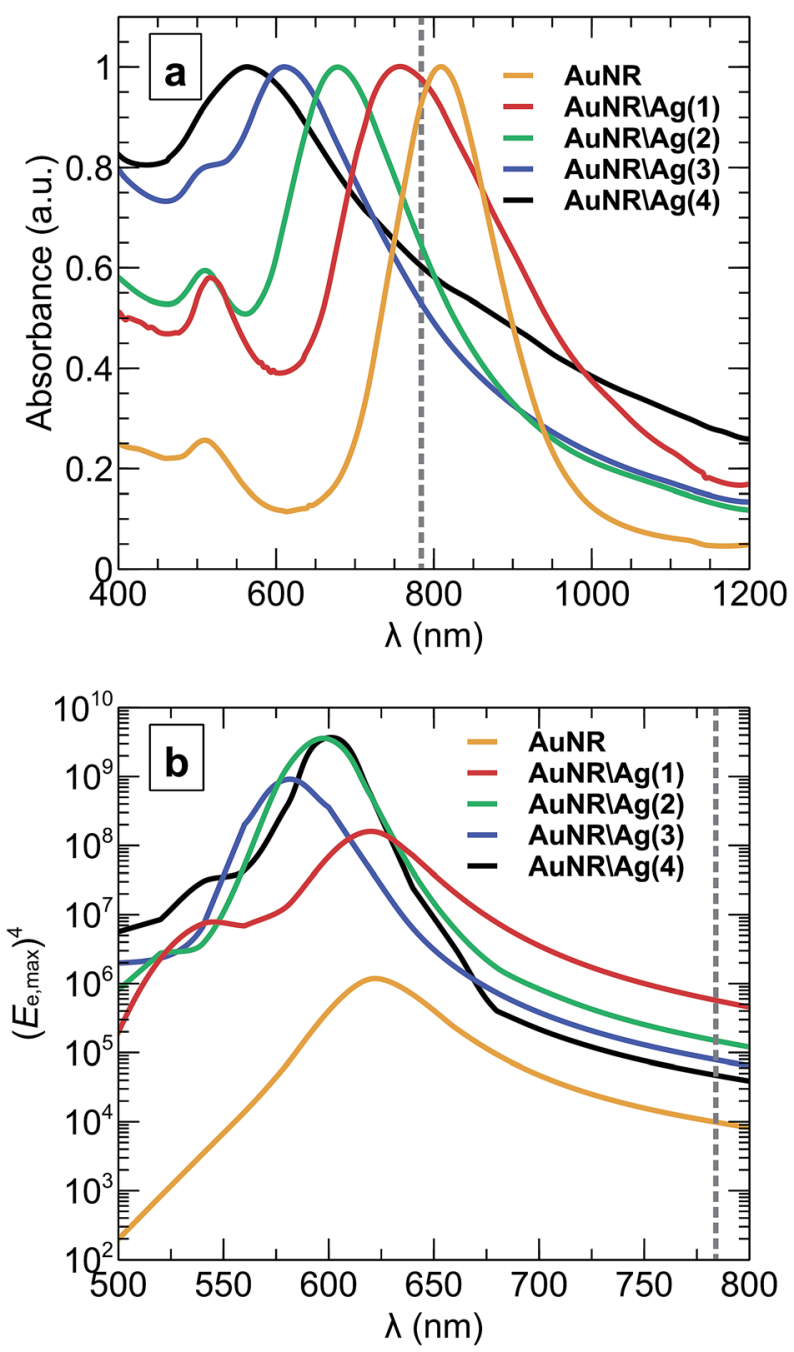

Fig. 3 (a) Absorbance spectra of colloidal suspensions with core gold nanorods coated with $0,1,2,3$ and $4 \mathrm{~nm}$ silver shells, obtained from the UV-vis-NIR spectroscopic experiment. (b) The fourth power of the maximum electric field enhancement, $\left(E_{e, m a x}\right)^{4}$, in the vicinity of the nanoparticle on top of a silica substrate. The grey dashed line marks the $784 \mathrm{~nm}$ wavelength of the Raman spectrometer.
$73 \mathrm{eV}$ is the bulk damping, and $\gamma_{\mathrm{rad}}=6.6 \times 10^{-7} \mathrm{eV} \mathrm{nm}^{-3}$ is the radiation damping. ${ }^{46}$ The dielectric function of silver was adopted from He and Zeng. ${ }^{47}$ The model was solved for about
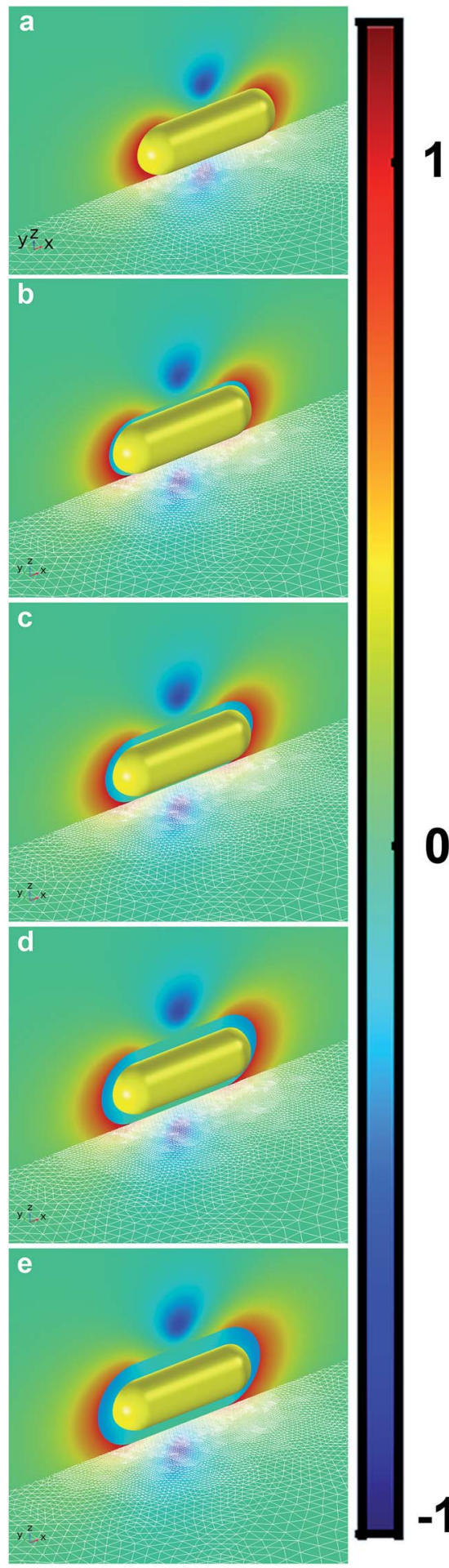

0

Fig. 4 The plots of the relative electric field enhancement, $\log _{10}\left(E_{\mathrm{e}}\right)$ in the vicinity of the AuNR and AuNR $\backslash$ Ags placed on a silica substrate at $784 \mathrm{~nm}$ excitation wavelength. (a) Bare gold nanorod (AuNR) with an aspect ratio of 3, (b) AuNR\Ag(1) with a $1 \mathrm{~nm}$ silver coating, (c) AuN$\mathrm{R} \backslash A g(2)$ with a $2 \mathrm{~nm}$ silver coating, (d) AuNR\Ag(3) with a $3 \mathrm{~nm}$ silver coating, and (e) AuNR\Ag(4) with a $4 \mathrm{~nm}$ silver coating. 
2.5 million degrees of freedom, with a requirement of $220 \mathrm{~Gb}$ of RAM.

\section{Results and discussion}

\subsection{Experimental results}

AuNR and AuNR $\backslash$ Ag Raman agents were synthesized using a modified seed-mediated method $^{6}$ which is based on the moderate reduction of gold and silver ions in the presence of detergent-like molecules (CTAB). Five types of the prepared nanomaterials were found to have unique spectral properties ideal for use as highly sensitive SERS Raman agents, especially for biomedical applications. ${ }^{2,6,48}$ Raman spectroscopy is one of the most effective techniques that can be applied non-destructively to study various kinds of medical disorders like cancer for instance, ${ }^{\mathbf{4 9 , 5 0}}$ as well as for the determination of trace and single molecular level analytes. ${ }^{\mathbf{5 1}}$

The size and shape of AuNR\Ags on top of a SERS Raman agent were obtained from HRTEM images, see Fig. $2 \mathrm{f}-\mathrm{j}$. The gold core rod was approximately $36 \pm 0.16 \mathrm{~nm}$ long and $12 \pm$ $0.13 \mathrm{~nm}$ wide, which corresponds to an aspect ratio of $\sim 3$. The silver coating thickness for each class of SERS agent was $\sim 1,2$, 3 , and $4 \mathrm{~nm}$ approximately. The elemental composition of the AuNRs and AuNR $\backslash$ Ags was also confirmed using X-ray photoelectron spectroscopy ${ }^{52}$ (see ESI document $\dagger$ ). The total surface charge (zeta-potential) for each species of the AuNRs and AuNR $\backslash$ Ags was determined using ZetaView ${ }^{\circledR}$, from Electrophoresis and Brownian Motion Video Analysis Laser Scattering Microscopy, see Table S2 ESI. $\dagger$
Fig. 3a shows the UV-vis-NIR spectra of the AuNRs and the AuNR $\backslash$ Ags with 1, 2, 3 and $4 \mathrm{~nm}$ silver shell thicknesses in colloidal solution. The addition of the silver shell blue shifts the longitudinal surface plasmon resonance wavelength from $810 \mathrm{~nm}$ to $580 \mathrm{~nm}$ (see Fig. 3a). The transverse plasmon resonance position also blue-shifted from $514 \mathrm{~nm}$ to $380 \mathrm{~nm}$ due to the silver shell. The blue-shifting of the longitudinal and transverse plasmon resonance spectra has two causes. The longitudinal surface plasmon resonance of a nanorod depends on its length-to-width aspect ratio. ${ }^{41}$ In contrast, the dielectric properties of the silver shell affect the blue-shifting transverse and longitudinal plasmon resonances. Therefore, changing a nanorod's aspect ratio from high to low will shift the surface plasmon resonance to lower wavelengths (see Fig. 3a), and varying the length of the nanorod will change the surface plasmon maximum absorption peak position (the extinction cross-section position and value).

The optical properties of AuNRs are highly dependent on the environment in the vicinity of the particle ${ }^{43}$ and, thus, will be different when in a homogeneous colloidal solution and when a single nanorod is on a substrate. Using the numerical model the optical properties of the nanoparticles in a colloidal solution (Fig. 3a) and on top of a silica substrate in air (Fig. 3b) were compared. Fig. 3b shows the fourth power of the maximum electric field enhancement, $\left(E_{\mathrm{e}, \max }\right)^{4}$, in the vicinity of a nanoparticle on a substrate calculated using the numerical model. As anticipated, the longitudinal near-field resonance of the electric field enhancement blue shifted by between $40 \mathrm{~nm}$ and $190 \mathrm{~nm}$. By comparing the optical properties of nanoparticles in a colloidal solution (Fig. 3a) and a nanoparticle on top of the

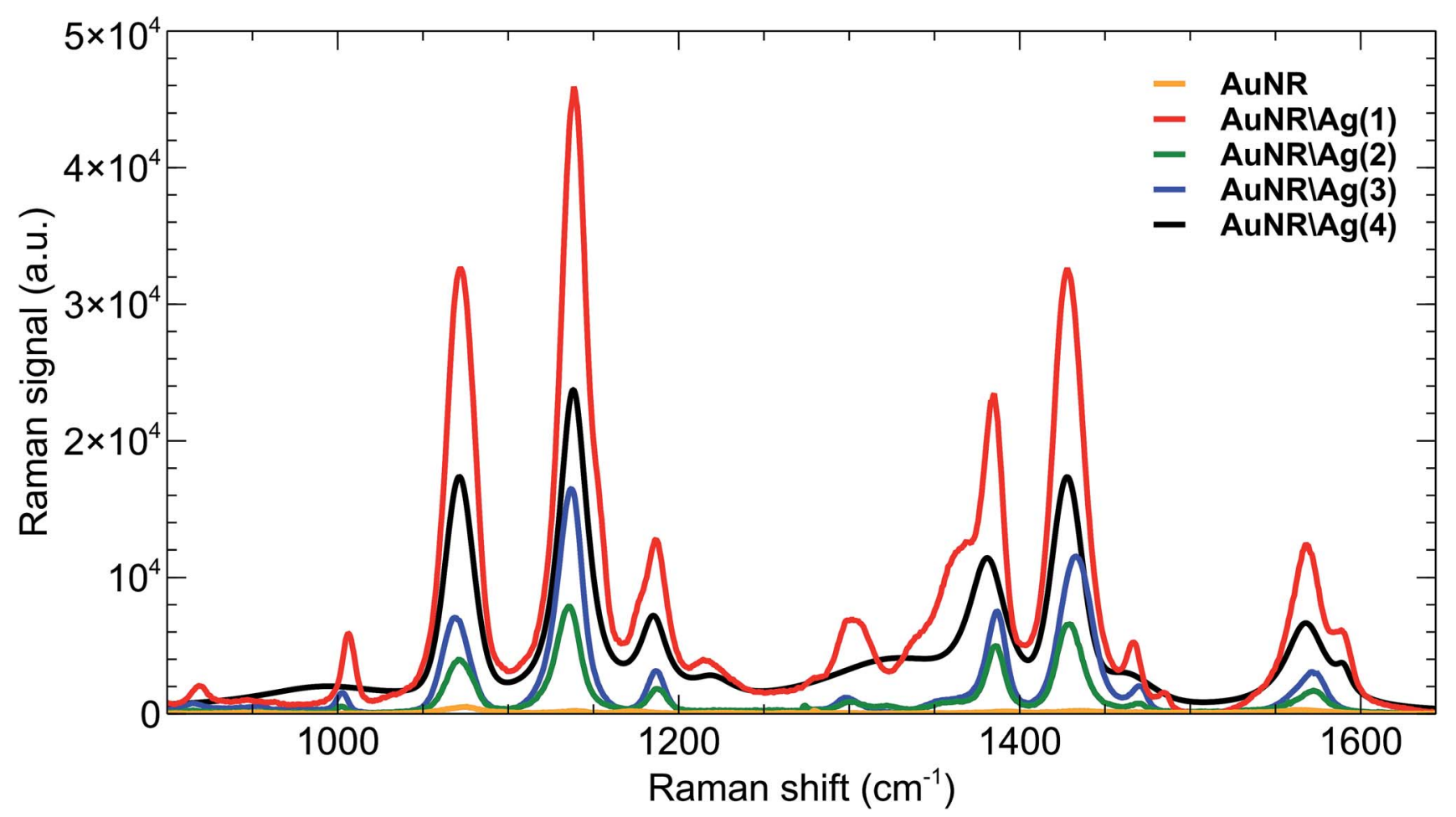

Fig. 5 SERS signals from Raman agents with bare gold nanorods (AuNRs) and gold nanorods with 1, 2, 3 and 4 nm silver thicknesses (AuNR\Ags). 
substrate in air (Fig. 3b), one can observe that although AuN$\mathrm{R} \backslash \mathrm{Ag}(1)$ with a $1 \mathrm{~nm}$ silver shell has longitudinal plasmon resonance close to the wavelength of Raman excitation at $784 \mathrm{~nm}$ (which is marked by the grey dashed line in Fig. 3a), all of the types of nanoparticles actually appear to be off-resonance when placed on a substrate. These results confirm the necessity of accounting for the environment of the nanoparticles ${ }^{43}$ when

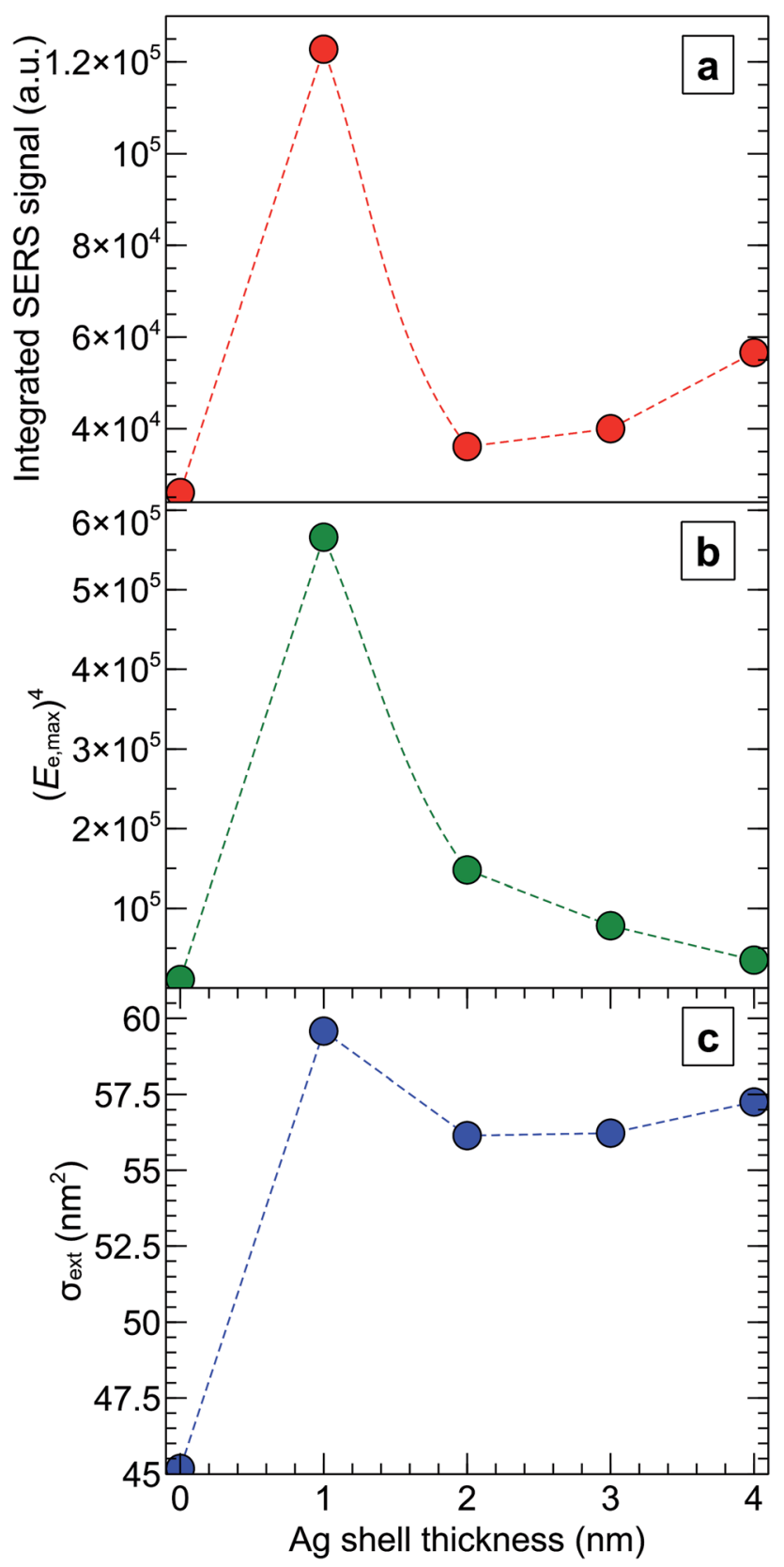

Fig. 6 Experimental SERS signals and numerical calculations of the field enhancements and extinction cross-sections of AuNRs and AuNR\Ags with different silver shell thicknesses at $784 \mathrm{~nm}$ wavelength. (a) SERS integrated signal recorded for each kind of SERS Raman agent using a Raman spectrometer for the peak at $1080 \mathrm{~cm}^{-1}$ (see Fig. 5). (b) The fourth power of the maximum electric field enhancement, $\left(E_{e, \max }\right)^{4}$, in the vicinity of a nanoparticle on top of the silica substrate. (c) The extinction cross-section, $\sigma_{\text {ext }}$, calculated for each type of nanoparticle on top of the substrate. working on the optimization of the SERS agents, rather then relying on the spectroscopic measurements of the bulk colloidal solution.

Fig. 4 shows the relative electric field enhancement, $E_{\mathrm{e}}$, in the vicinity of a nanoparticle on top of the silica substrate at $784 \mathrm{~nm}$ incident wavelength on a log-scale, as well as the surface mesh discretization of the silica substrate.

The integrated SERS signal for each type of Raman agent shows that the maximum surface enhanced Raman signal was obtained from a sample of AuNR $\backslash \mathrm{Ag}$ coated with a $1 \mathrm{~nm}$ silver shell, as illustrated in Fig. 5. The experimental results were compared against numerical predictions. A simulation of the response of a single nanoparticle on top of the silica substrate in air to a circularly polarized electric field at $784 \mathrm{~nm}$ wavelength also showed that AuNR $\backslash$ Ag with $1 \mathrm{~nm}$ silver thickness exhibited the highest maximum electric field enhancement, $E_{\text {e,max }}$, and extinction cross-section, $\sigma_{\text {ext }}$, as shown in Fig. $6 \mathrm{~b}$ and c. However, the model predicted that the maximum electric field enhancement for AuNR $\backslash \mathrm{Ag}$ with a $4 \mathrm{~nm}$ silver shell should be lower than the maximum electric field enhancements for AuNR $\backslash$ Ag with $2 \mathrm{~nm}$ and $3 \mathrm{~nm}$ silver shells, which was not seen experimentally. The calculated extinction cross-section of the AuNR $\backslash \mathrm{Ag}$ with a $4 \mathrm{~nm}$ silver shell was higher than the extinction cross-sections of the AuNR\Ag with $2 \mathrm{~nm}$ and $3 \mathrm{~nm}$ silver shells, which better correlates with the experimental SERS signal results.

Generally, both the experimental and simulation results were in agreement and showed that the $1 \mathrm{~nm}$ silver-coated gold nanorods SERS Raman agent generated, at $784 \mathrm{~nm}$, the highest SERS signal (Fig. 6a) and the highest electric field enhancement and extinction cross-section (Fig. $6 \mathrm{~b}$ and c). Based on numerical simulation of the single nanoparticle on a substrate (Fig. 3b), the similar qualitative picture of the SERS signal enhancement versus different silver shell thickness, as in Fig. 6a, should hold true for wavelengths from $680 \mathrm{~nm}$ to $800 \mathrm{~nm}$. Moreover, the enhancement of the SERS signal will be different for different studies of the nanoparticles, when the SERS excitation wavelength will be in the range of $500 \mathrm{~nm}$ to $650 \mathrm{~nm}$, where AuN$\mathrm{R} \backslash$ Ags exhibit LSPR (Fig. 3b).

Raman enhancement factors for the gold nanorod and silver coated gold nanorods were calculated based on the $1080 \mathrm{~cm}^{-1}$ peak. All peaks were baselined and the integrated SERS signals were estimated using LabSpec software (Table 1).

Table 1 Enhancement factors for: PATP ( $p$-aminothiophenol), AuNR and AuNR $\backslash$ Ags with different silver shell thicknesses at $784 \mathrm{~nm}$ wavelength

\begin{tabular}{lll}
\hline Nanorods & $\begin{array}{l}\text { SERS integrated } \\
\text { signal (a.u.) }\end{array}$ & $\begin{array}{l}\text { Enhancement } \\
\text { factor }\end{array}$ \\
\hline PATP & 582 & 1 \\
$\operatorname{AuNR}$ & 67847 & 117 \\
$\operatorname{AuNR} \backslash \operatorname{Ag}(1)$ & 580648 & 998 \\
$\operatorname{AuNR} \backslash \operatorname{Ag}(2)$ & 175364 & 301 \\
$\operatorname{AuNR} \backslash \operatorname{Ag}(3)$ & 384545 & 661 \\
$\operatorname{AuNR} \backslash \operatorname{Ag}(4)$ & 427870 & 735
\end{tabular}




\section{Conclusions}

Experimental and simulation data for five kinds of goldnanorod-based SERS agents were collected to assess their potential for biomedical applications. A chemical-based assay was used to synthesize the nanomaterials. Each type was characterized using a high-resolution transmission electron microscope and X-ray photoelectron spectroscopy. Moreover, optical absorbance was also recorded for each type of colloidal nanoparticle to determine the wavelength of the longitudinal surface plasmon resonance. Additionally, a numerical model of a gold nanoparticle on top of a silica substrate in air exposed to $784 \mathrm{~nm}$ wavelength circularly polarized light was solved for each type of nanomaterial in a range of wavelengths. The numerical model accurately predicted the experimental dependence of the SERS intensity variation with $\mathrm{Ag}$ thickness and, therefore, precisely predicted the optical properties of the nanoparticles that were generated in the experiment. All of the AuNR $\backslash$ Ags produced a superior surface-enhanced Raman spectra in comparison to the bare AuNRs. However, under $784 \mathrm{~nm}$ laser excitation, the AuNR $\backslash$ Ags coated with a $1 \mathrm{~nm}$ silver shell produced the highest enhancement.

\section{Conflicts of interest}

The authors declare that there are no conflicts of interest.

\section{Acknowledgements}

This work was supported by grants from the Center for Advanced Surface Engineering, under the National Science Foundation Grant No. 1457888 and the Arkansas EPSCoR Program, ASSET III.

\section{References}

1 S. Abalde-Cela, P. Aldeanueva-Potel, C. Mateo-Mateo,

L. Rodríguez-Lorenzo, R. A. Alvarez-Puebla and

L. M. Liz-Marzán, J. R. Soc., Interface, 2010, S435-S450.

2 Z. A. Nima, A. Biswas, I. S. Bayer, F. D. Hardcastle, D. Perry, A. Ghosh, E. Dervishi and A. S. Biris, Drug Metab. Rev., 2014, 46, 155-175.

3 M. Vendrell, K. K. Maiti, K. Dhaliwal and Y.-T. Chang, Trends Biotechnol., 2013, 31, 249-257.

4 H. Abramczyk and B. Brozek-Pluska, Chem. Rev., 2013, 113, 5766-5781.

5 X. Shen, T. J. Cui, D. Martin-Cano and F. J. Garcia-Vidal, Proc. Natl. Acad. Sci. U. S. A., 2013, 110, 40-45.

6 Z. A. Nima, M. Mahmood, Y. Xu, T. Mustafa, F. Watanabe, D. A. Nedosekin, M. A. Juratli, T. Fahmi, E. I. Galanzha, J. P. Nolan, A. G. Basnakian, V. P. Zharov and A. S. Biris, Sci. Rep., 2014, 4, 4752.

7 K. Kneipp, A. S. Haka, H. Kneipp, K. Badizadegan, N. Yoshizawa, C. Boone, K. E. Shafer-Peltier, J. T. Motz, R. R. Dasari and M. S. Feld, Appl. Spectrosc., 2002, 56, 150154.

8 S. Nie and S. R. Emory, Science, 1997, 275, 1102-1106.
9 S. Nie, Y. Xing, G. J. Kim and J. W. Simons, Annu. Rev. Biomed. Eng., 2007, 9, 257-288.

10 W. L. Barnes, A. Dereux and T. W. Ebbesen, Nature, 2003, 424, 824-830.

11 A. V. Zayats, I. I. Smolyaninov and A. A. Maradudin, Phys. Rep., 2005, 408, 131-314.

12 J. Pendry, Science, 1999, 285, 1687-1688.

13 A. I. Csurgay and W. Porod, Int. J. Circ. Theor. App., 2004, 32, 339-361.

14 P. Berini and I. De Leon, Nat. Photonics, 2012, 6, 16-24.

15 S. Kawata, Y. Inouye and P. Verma, Nat. Photonics, 2009, 3, 388-394.

16 J. N. Anker, W. P. Hall, O. Lyandres, N. C. Shah, J. Zhao and R. P. Van Duyne, Nat. Mater., 2008, 7, 442-453.

17 Z. A. Nima, M. H. Lahiani, F. Watanabe, Y. Xu, M. V. Khodakovskaya and A. S. Biris, $R S C A d v$., 2014, 4, 64985-64993.

18 B. Sharma, M. F. Cardinal, S. L. Kleinman, N. G. Greeneltch, R. R. Frontiera, M. G. Blaber, G. C. Schatz and R. P. V. Duyne, MRS Bull., 2013, 38, 615-624.

19 M. J. Banholzer, J. E. Millstone, L. Qin and C. A. Mirkin, Chem. Soc. Rev., 2008, 37, 885-897.

20 A. J. Haes and R. P. V. Duyne, Expert Rev. Mol. Diagn., 2004, 4, 527-537.

21 S. L. Kleinman, R. R. Frontiera, A.-I. Henry, J. A. Dieringer and R. P. Van Duyne, Phys. Chem. Chem. Phys., 2013, 15, 21-36.

22 H. K. Lee, Y. H. Lee, Q. Zhang, I. Y. Phang, J. M. R. Tan, Y. Cui and X. Y. Ling, ACS Appl. Mater. Interfaces, 2013, 5, 1140911418.

23 M. Fan and A. G. Brolo, Phys. Chem. Chem. Phys., 2009, 11, 7381-7389.

24 J. Ma, Z. Hu, W. Wang, X. Wang, Q. Wu and Z. Yuan, ACS Appl. Mater. Interfaces, 2017, 9, 16767-16777.

25 Y. Tanoue, K. Sugawa, T. Yamamuro and T. Akiyama, Phys. Chem. Chem. Phys., 2013, 15, 15802-15805.

26 D. L. Jeanmaire and R. P. V. Duyne, J. Electroanal. Chem. Interfacial Electrochem., 1977, 84, 1-20.

27 J. A. Creighton, C. G. Blatchford and M. G. Albrecht, J. Chem. Soc., Faraday Trans. 2, 1979, 75, 790-798.

28 P. C. Lee and D. Meisel, J. Phys. Chem., 1982, 86, 3391-3395. 29 M. Jin, H. van Wolferen, H. Wormeester, A. van den Berg and E. T. Carlen, Nanoscale, 2012, 4, 4712-4718.

30 Q. Yu, S. Braswell, B. Christin, J. Xu, P. M. Wallace, H. Gong and D. Kaminsky, Nanotechnology, 2010, 21, 355301.

31 R. P. V. Duyne, J. C. Hulteen and D. A. Treichel, J. Chem. Phys., 1993, 99, 2101-2115.

32 J. R. Anema, J.-F. Li, Z.-L. Yang, B. Ren and Z.-Q. Tian, Annu. Rev. Anal. Chem., 2011, 4, 129-150.

33 S. J. Barcelo, A. Kim, W. Wu and Z. Li, ACS Nano, 2012, 6, 6446-6452.

34 K. D. Osberg, M. Rycenga, N. Harris, A. L. Schmucker, M. R. Langille, G. C. Schatz and C. A. Mirkin, Nano Lett., 2012, 12, 3828-3832.

35 K.-K. Liu, S. Tadepalli, G. Kumari, P. Banerjee, L. Tian, P. K. Jain and S. Singamaneni, J. Phys. Chem. C, 2016, 120, 16899-16906. 
36 M. Lin, Y. Wang, X. Sun, W. Wang and L. Chen, ACS Appl. Mater. Interfaces, 2015, 7, 7516-7525.

37 T. Bai, J. Sun, R. Che, L. Xu, C. Yin, Z. Guo and N. Gu, ACS Appl. Mater. Interfaces, 2014, 6, 3331-3340.

38 Y. Zhang, Z. Wang, L. Wu, Y. Pei, P. Chen and Y. Cui, Analyst, 2014, 139, 5148-5154.

39 R. Contreras-Caceres, C. Dawson, P. Formanek, D. Fischer, F. Simon, A. Janke, P. Uhlmann and M. Stamm, Chem. Mater., 2013, 25, 158-169.

40 B. N. Khlebtsov, V. A. Khanadeev, M. Y. Tsvetkov, V. N. Bagratashvili and N. G. Khlebtsov, J. Phys. Chem. C, 2013, 117, 23162-23171.

41 B. Nikoobakht and M. A. El-Sayed, Chem. Mater., 2003, 15, 1957-1962.

42 https://www.comsol.com/model/scatterer-on-a-substrate14699.

43 Y. Davletshin, A. Lombardi, M. Cardinal, V. Juvé, A. Crut, P. Maioli, L. Liz-Marzán, F. Vallée, N. Del Fatti and J. Kumaradas, ACS Nano, 2012, 6, 8183-8193.
44 P. Johnson and R. Christy, Phys. Rev. B: Solid State, 1972, 6, 4370-4379.

45 V. Juvé, M. F. Cardinal, A. Lombardi, A. Crut, P. Maioli, J. Pérez-Juste, L. M. Liz-Marzán, N. Del Fatti and F. Vallée, Nano Lett., 2013, 13, 2234-2240.

46 Z. Li, W. Mao, M. S. Devadas and G. V. Hartland, Nano Lett., 2015, 15, 7731-7735.

47 Y. He and T. Zeng, J. Phys. Chem. C, 2010, 114, 18023-18030.

48 S. A. Maier, Plasmonics: Fundamentals and Applications, Springer, US, 1st edn, 2007.

49 S. Lee, H. Chon, J. Lee, J. Ko, B. H. Chung, D. W. Lim and J. Choo, Biosens. Bioelectron., 2014, 51, 238-243.

50 J. P. Nolan, E. Duggan, E. Liu, D. Condello, I. Dave and S. A. Stoner, Methods, 2012, 57, 272-279.

51 K. Kneipp, Y. Wang, H. Kneipp, L. T. Perelman, I. Itzkan, R. R. Dasari and M. S. Feld, Phys. Rev. Lett., 1997, 78, 1667-1670.

52 F. Watanabe, Z. A. Nima, T. Honda, M. Mitsuhara, M. Nishida and A. S. Biris, Nanotechnology, 2017, 28, 025704. 